\title{
Electrocatalytic oxidation enhancement at the surface of InGaN films and nanostructures grown directly on $\mathrm{Si}(111)$
}

\author{
Paul E.D. Soto Rodriguez ，Veronica Calderon Nash , Pavel Aseev , Victor J. Gómez , Praveen Kumar , \\ Naveed Ul Hassan Alvi , Alfredo Sánchez , Reynaldo Villalonga , José M. Pingarrón , Richard Nötzel
}

\begin{abstract}
A B S T R A C T
Pronounced electrocatalytic oxidation enhancement at the surface of InGaN layers and nanostructures directly grown on Si by plasma-assisted molecular beam epitaxy is demonstrated. The oxidation enhancement, probed with the ferro/ferricyanide redox couple increases with In content and proximity of nanostructure surfaces and sidewalls to the c-plane. This is attributed to the corresponding increase of the density of intrinsic positively charged surface donors promoting electron transfer. Strongest enhancement is for c-plane InGaN layers functionalized with InN quantum dots (QDs). These results explain the excellent performance of our InN/InGraN QD biosensors and water splitting electrodes for further boosting efficiency.
\end{abstract}

\section{Introduction}

III-nitride semiconductors are of high interest for optoelectronics and high-power electronics [1-3]. Recently, the application fields were extended to electrochemical electrodes. InGaN is the first choice for these applications due to its excellent intrinsic properties [4] (widest bandgap tunability, largest carrier mobility, high chemical stability). Therefore, understanding of the electrochemical performance is essential, currently lacking for the whole In composition range, especially for In-rich InGaN with the optimum near-infrared bandgap for solar light absorption.

We have recently demonstrated unprecedented performance of InN quantum dots (QDs) grown on planar In-rich InGaN layers on c-plane GaN/Sapphire templates as biosensor transducers and photoelectrochemical electrodes $[5,6]$. This was attributed to the high density of positively charged surface donor states $\left(\rho^{+}\right.$SD $)$of cplane InGaN, increasing with In content above $40-50 \%$ up to $2-3$ $10^{13} \mathrm{~cm}^{-2}$ for pure InN, together with the zero-dimensional QD electronic properties. These $\rho^{+}$SD facilitate oxidation by attraction of electrons. For planar layers they are uniformly compensated by electron surface accumulation. Uncompensated surface charge enhancing the electron transfer arises on the QDs as sufficient electrons (equivalent to the number of donors on the QDs) cannot enter the QDs due to the Pauli Exclusion Principle. Ultimately, we have grown InGaN layers over the entire composition range on $\mathrm{Si}(111)$ together with InN QDs on top [7, 8], allowing integration with Si technology and reducing cost. Moreover, we have studied the growth of InGaN nanowall networks (NWNs) on $\mathrm{Si}(111)$ for water splitting $[9,10]$.

Here we present the electrochemical properties of InGaN layers over the entire composition range, and of In-rich InGaN NWNs and nanocolumns (NCs) on Si. The electrochemical characterization was performed by cyclic voltammetry (CV) using the ferro/ferricyanide redox probe. Despite enlarged surface area for the nanostructured InGaN layers, the electrocatalytic activity is enhanced for polar c-plane InGaN layers with increasing In content as for In-rich c-plane InGaN layers with InN QDs on top; attributed to the increasingly largest $\rho^{+}$SD and zero-dimensional QD properties. Weak or no electrocatalytic activity enhancement occurs for the NWN and NCs. They are bound by semi-polar planes or non-polar m-planes where reduced density or absence of $\rho^{+}$SD is stated [11-15]. Therefore, balance is understood between electrocatalytic activity of a certain surface and the surface area to optimize electrochemical performance: the former governing the energetics, i.e., the possibility of the chemical reaction to take place and the latter governing the amount of a possible reaction. 
Table 1

Growth temperatures, V-III flux ratios, In and Ga fluxes, In contents and bandgap energies for all samples.

\begin{tabular}{|c|c|c|c|c|c|c|}
\hline Sample & $\begin{array}{l}\text { Growth temperature } \\
\left({ }^{\circ} \mathrm{C}\right)\end{array}$ & $\begin{array}{l}\text { V-III flux } \\
\text { ratio }\end{array}$ & $\begin{array}{l}\text { In flux } \\
\left(10^{13} \text { atoms } \mathrm{s}^{-1} \mathrm{~cm}^{-2}\right)\end{array}$ & $\begin{array}{l}\text { Ga flux } \\
\left(10^{13} \text { atoms s} ~^{-1} \mathrm{~cm}^{-2}\right)\end{array}$ & $\begin{array}{l}\text { In content } \\
(\%)\end{array}$ & $\left.\mathrm{E}_{\mathrm{g}} \mathrm{eV}\right)$ \\
\hline M1 & 450 & 1.3 & 3.32 & 52.1 & 6 & 3.09 \\
\hline M2 & 400 & 1.37 & 18.4 & 34.2 & 35 & 1.89 \\
\hline M3 & 450 & 1.58 & 33.3 & 12.3 & 73 & 0.94 \\
\hline M4 & 450 & 1.69 & 37.5 & 5.11 & 88 & 0.76 \\
\hline M5 & 485 & 2 & 21.6 & 14.4 & 60 & 1.18 \\
\hline M6 & 400 & 4.2 & 11.5 & 5.66 & 67 & 1.04 \\
\hline
\end{tabular}

\section{Experimental}

Growth was performed by plasma-assisted molecular beam epitaxy (PA-MBE). 1-inch p-type Si(111) wafers were degassed $4 \mathrm{~h}$ at $450^{\circ} \mathrm{C}$ in the introduction chamber and $30 \mathrm{~min}$ at $850^{\circ} \mathrm{C}$ in the growth chamber for native oxide removal. The $\mathrm{Si}(111)$ surface was then nitrided at $850{ }^{\circ} \mathrm{C}$ for 5 min under $7.16 \times 10^{14}$ atoms $/ \mathrm{s} \mathrm{cm}^{2}$ active $\mathrm{N}_{2}$ flux ( $0.9 \mathrm{sccm}$ molecular $\mathrm{N}_{2}$ flow and $350 \mathrm{~W}$ radio-frequency power). After cooling down, InGaN was grown for $1 \mathrm{~h}$ for all samples. The In content was adjusted by the In and $\mathrm{Ga}$ fluxes. The active $\mathrm{N}_{2}$ flux was constant during nitridation and growth. The growth temperatures, V-III flux ratios, and In and Ga fluxes are listed in Table 1.
The InGaN morphology and thickness/side view were investigated by scanning electron microscopy (SEM). The In content was determined by reciprocal space maps with a high-resolution X-ray diffractometer. The bandgap was determined from the In content using a bowing parameter of $2.5 \mathrm{eV}$ [7]. In contents and bandgap energies $\mathrm{E}_{\mathrm{g}}$ are also listed in Table 1.

Samples M1-M4, grown under slightly N-rich conditions with V-III flux ratio around 1.5 are compact layers with increasing In content, distinct c-plane island structure (see SEM images in Fig. 1 (a-d)), and $\sim 450 \mathrm{~nm}$ thickness. For sample M5, the V-III flux ratio was increased to 2 and the growth temperature to $485^{\circ} \mathrm{C}$. For sample M6 the V-III flux ratio was adjusted to 4 and the growth temperature to $400{ }^{\circ} \mathrm{C}$.

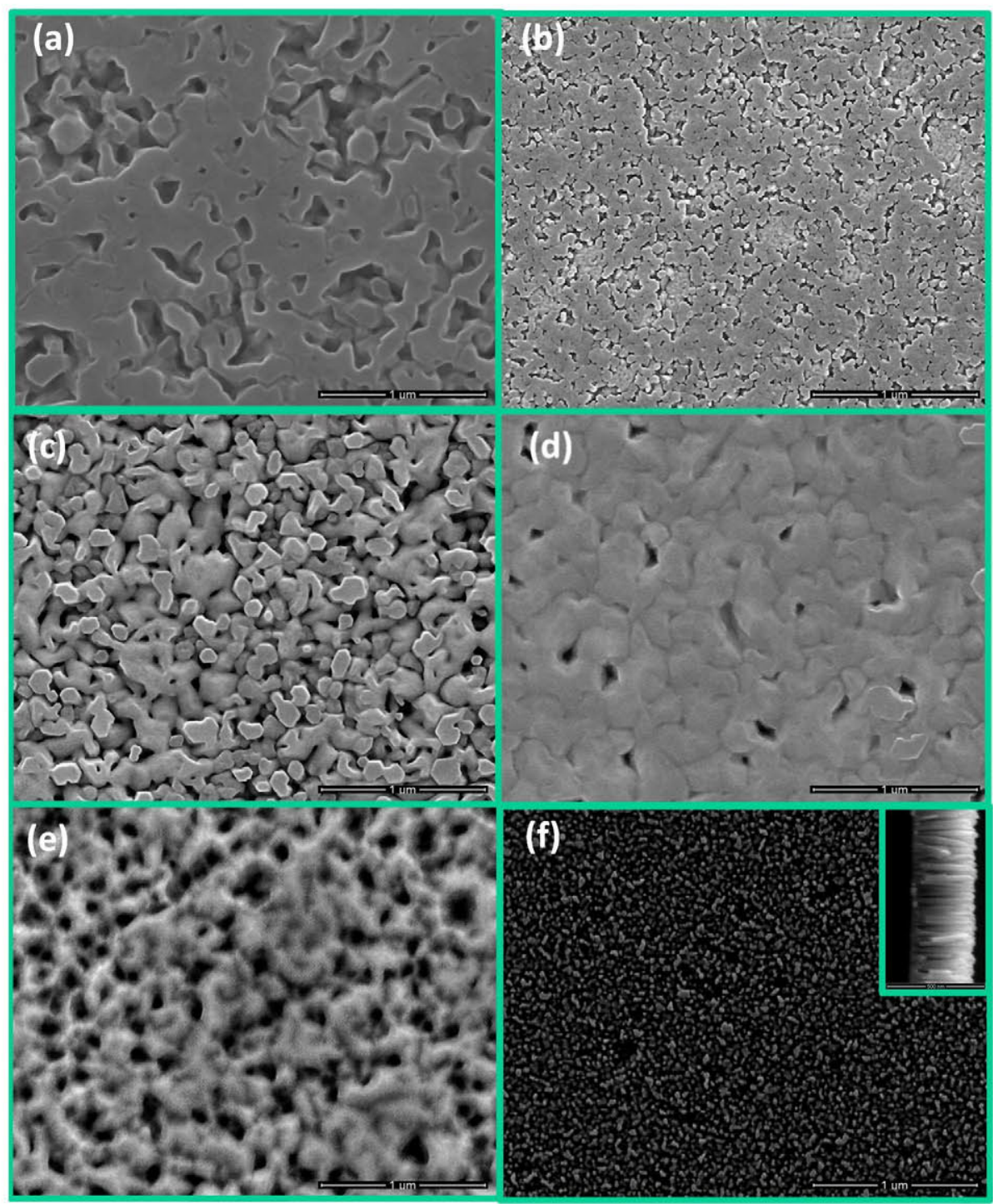

Fig. 1. (a)-(f) Plan-view SEM images of samples M1-M6. Inset in (f): cross-section of M6. 
Sample M5 (SEM image in Fig. 1(e)) exhibits a NWN and sample M6 NCs (plan-view and cross-sectional SEM images in Fig. 1(f)). Additionally, two samples were grown as M3 and M5 with InN QDs on top. The QDs were formed by supplying the In flux of M3 for $10 \mathrm{~s}$ for sample 'M3 plus InN QDs' and after In flux adjustment for sample 'M5 plus InN QDs'.

Silver paste contacts were deposited. The contacts are Ohmic for the larger In contents due to the high defect-induced n-type bulk conductivity of InGaN. The contacts were placed on the sample surfaces not in contact with the electrolyte. For the NCs this implies contact with the $\mathrm{p}$-Si substrate which is also in contact with the electrolyte. Independent measurements, however, showed that the p-Si substrate is not electrochemically active. Further, the contact between In-rich InGaN and $\mathrm{p}-\mathrm{Si}$ is ohmic [7], guaranteeing a valid measurement of the electrochemical properties also for the NCs. A three-electrode electrochemical cell was used for the CV measurements with InGaN working electrode, Pt auxiliary electrode and $\mathrm{Ag} / \mathrm{AgCl}$ reference electrode connected to a potentiostat. The sample area exposed to the electrolyte was $7.5 \mathrm{~mm}^{2}$. The electrocatalytic properties of the InGaN samples were probed with the ferro/ferricyanide redox couple with $5 \mathrm{mM}$ equimolar concentration and $0.1 \mathrm{M} \mathrm{KCl}$ in aqueous solution as supporting electrolyte. The scan rate was $25 \mathrm{mVs}^{-1}$. The electrode potential $(E)$ was scanned from positive to negative potentials and back over ranges to fully develop the characteristic shapes, limited to avoid other reactions.

\section{Results and discussion}

3.1. Electrocatalytic characterization of InGaN films with different In-content

Fig. 2 (A-D), presents CV measurements for samples M1-M4. For all CV measurements the faradaic anodic and cathodic current peaks are due to the ferro/ferricyanide redox reaction, which is the established probe to characterize electrochemical electrodes due to the fast and reversible electron transfer: For the InGaN layers and nanostructures no contribution to the current is found due to the $\mathrm{KCl}$ aqueous electrolyte. Only for the c-plane InN QDs (Fig. 3, discussed below) weak features are observed at low anodic and cathodic potentials, whose origin is not within the scope of the present study. Moreover, there is no chemical reaction of the InGaN electrodes, as demonstrated by control experiments in $0.1 \mathrm{M} \mathrm{KCl}$ solutions. Repeated CV measurements reveal long time stability with unchanged electrochemical surface properties (data not shown) and there are no changes of other surface properties (morphological or optical) which are in line with the well known chemical stability of InGaN.

Sample M1 does not show clear anodic or pronounced cathodic current peaks, probably due to the wide InGaN bandgap and higher Ohmic resistance, shifting the anodic and cathodic peaks to potential values not recorded in this experiment. The anodic peak develops and shifts to a less positive $E$ upon In content increase, most clear for samples M2 to M3; less evident for samples M3 to M4. Samples M3 and M4 may have an InN like surface due to In surface accumulation/segregation. Also, a cathodic current peak develops for samples M2 and M3 and becomes suppressed for sample M4 showing an enhancement of catalytic oxidation with increasing In content due to the presence of the intrinsic $\rho^{+}{ }_{\text {SD }}$ with increasing density. This matches with the symmetric CV curve for sample $M 2$, i.e., quasi-reversible oxidation and reduction more like for metallic electrodes: The $\rho^{+}{ }_{\text {SD }}$ decreases with decreasing In content. Below around $40 \%$ In content the surface states move from the conduction band into the bandgap and, hence, change from donor-like to acceptor-like, causing a transition from electron accumulation to electron depletion [16]. Therefore, M2 is a common highlydoped n-type semiconductor behaving similar to metals. The lower current for sample M3 shows the largest electrochemical activity of
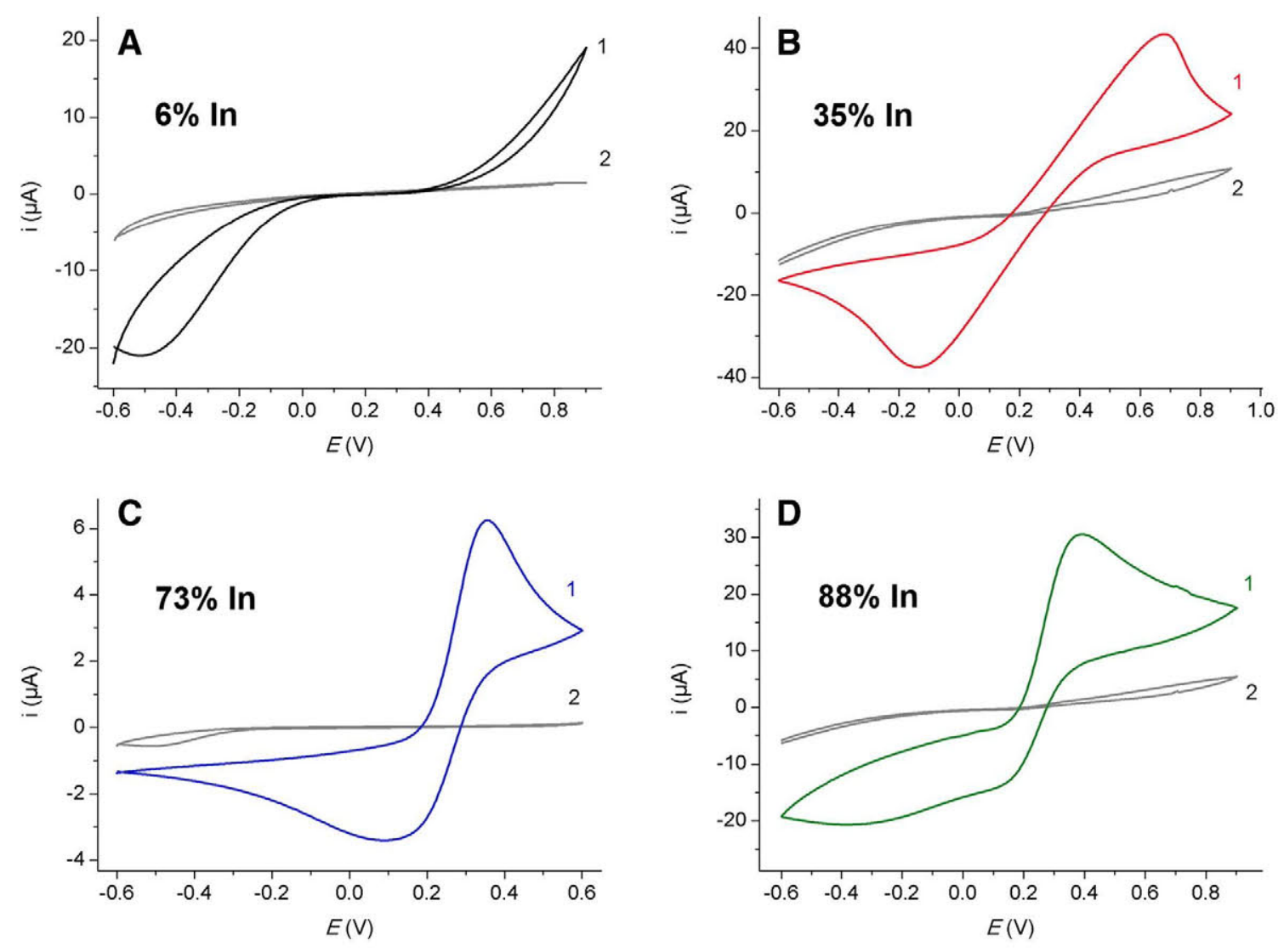

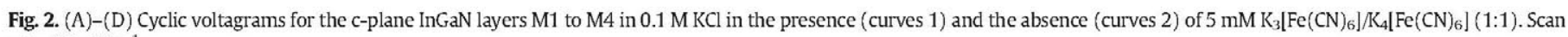
rate: $25 \mathrm{mVs}^{-1}$. 

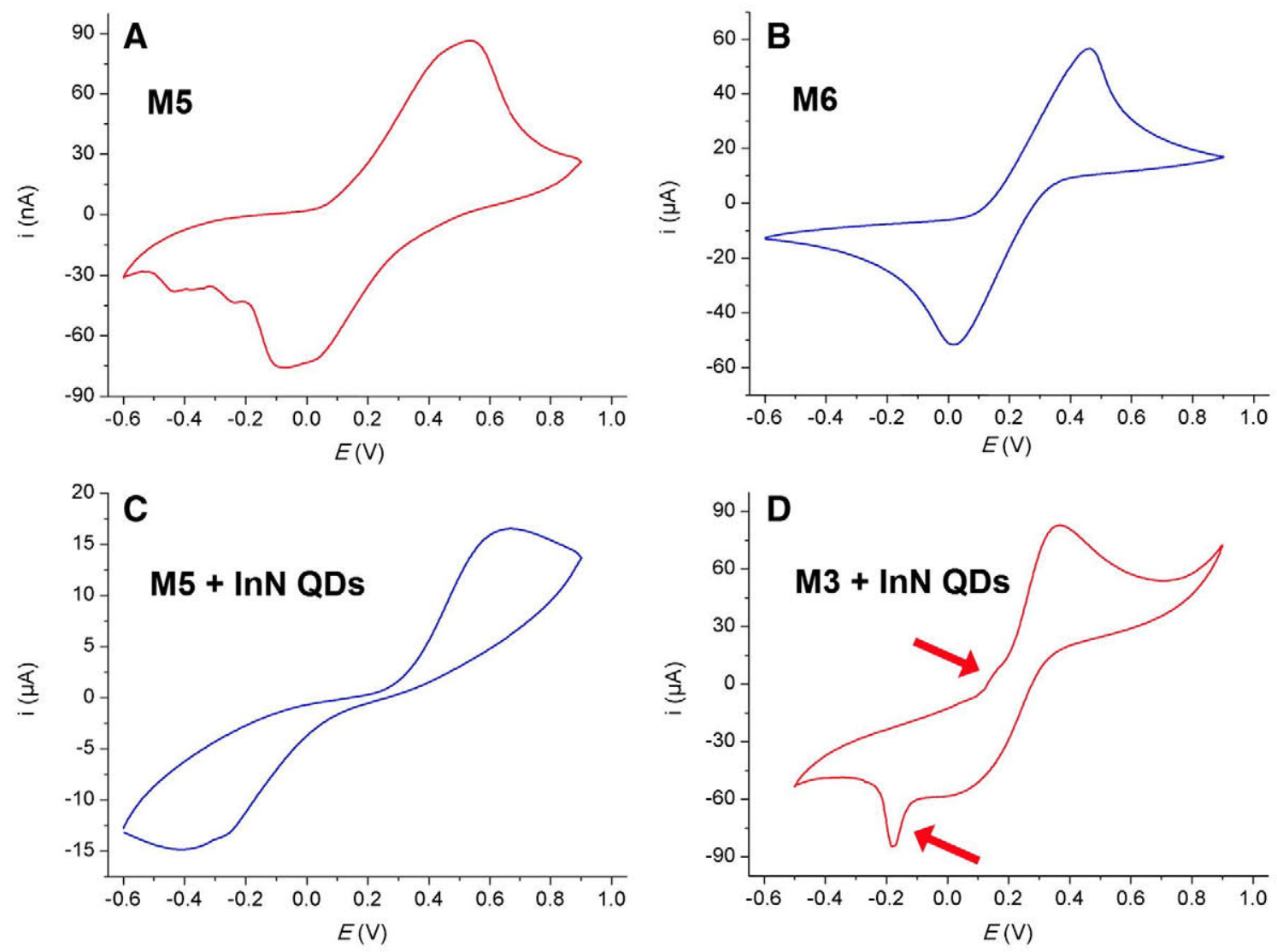

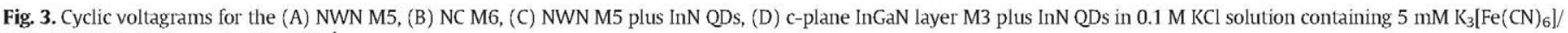
$\mathrm{K}_{4}\left[\mathrm{Fe}(\mathrm{CN})_{6}\right](1: 1)$. Scan rate: $25 \mathrm{mVs}^{-1}$.

the c-plane due to the highest $\rho^{+}{ }_{\mathrm{SD}}$ because its portion is the lowest for M3 as observed in the SEM image in Fig. 1(c). This becomes evident when discussing the NWN and NCs with surfaces inclined towards the c-plane of reduced or no electrocatalytic activity.

\subsection{Electrocatalytic characterization of InGaN NWN and NCS}

Fig. 3 (A, B) depicts the cyclic voltagrams of the NWN and NCs of samples M5 and M6. The $E$ of their anodic current peaks is consistent with those of samples M2-M4 for the In contents between 60 and $70 \%$ together with the quasi-reversible oxidation and reduction for these In contents. The peak currents are lowest for the NWN, exhibiting the smallest portion of active c-plane of all samples. Combining all results shows that the $E$ of the anodic current peak is given by the In content while the peak current is governed by the area of active c-plane - consistently referring back to the low current for sample M3.

For the NCs, the portion of active c-plane on top is still relevant leading to the high current. Despite the large surface area of the m-plane sidewalls the current is comparable to that of samples M2 and M4. Further, the $E$ of the anodic current peak agrees with that of the c-plane InGaN layers. This means that the $m$-planes are not or negligibly participating in the electrochemical reaction indicating the absence or low $\rho^{+}$SD are requiring a larger $E$.

\subsection{Effect of InN QDS on NWN and InGaN film}

Finally, the effects of the InN QDs for the NWN, sample 'M5 plus InN QDs', and for the c-plane InGaN layer with 73\% In content, sample 'M3 plus InN QDs',are shown in Fig. 3 (C, D). A clear enhancement of the overall currents indicates the QDs as active sites; notice the different current scale in the graphs. For the NWN, however, the $E$ of the anodic and cathodic current peaks is enlarged. This suggests that the QDs activate the semi-polar sidewalls of the NWN with lower $\rho^{+}$, thus, requiring a larger $E$. The anodic peak current of sample 'M3 plus InN QDs' is enhanced staying at a low $E$ while the cathodic current peak is suppressed (the weak features marked by arrows are due to the electrolyte). The anodic peak current of sample M3 is the highest. This confirms that the combination of the highest $\rho^{+}$SD for c-plane InN with the zero-dimensional QD electronic properties leads to the strongest increase of catalytic oxidation activity as observed in the biosensor and water splitting experiments $[5,6]$.

\section{Conclusions}

We have shown pronounced electrocatalytic oxidation enhancement at the surface of InGaN films and nanostructures directly grown on $\mathrm{Si}(111)$ by PA-MBE with an increasing In content and proximity of surface and nanostructure sidewalls to the c-plane. This was attributed to the increase of $\rho^{+}$SD promoting electron transfer. Best results are for c-plane In-rich InGaN layers functionalized with InN QDs, attributed to the highest $\rho^{+}{ }_{S D}$ together with the zero-dimensional electronic QD properties inducing uncompensated surface charge. These results are important for further boosting the efficiency of InN/InGaN QDs based biosensors and water-splitting, finding an optimum balance between catalytic efficiency driving the reaction and surface area increasing the amount.

\section{Notes}

The authors declare no competing financial interests. 


\section{Acknowledgement}

This work was supported by the Spanish Ministry of Economy and Competitiveness through the grant CTQ2014-58989-P.

\section{References}

[1] S. Nakamura, The roles of structural imperfections in InGaN-based blue lightemitting diodes and laser diodes, Science 281 (1998) 956-961.

[2] K. Okamoto, I. Niki, A. Shvartser, Y. Narukawa, T. Mukai, A. Scherer, Surface-plasmon-enhanced light emitters based on InGaN quantum wells, Nat. Mater. 3 (2004) 601-605.

[3] F.A. Ponce, D.P. Bour, Nitride-based semiconductors for blue and green lightemitting devices, Nature 386 (1997) 351-359.

[4] J. Wu, When group-III nitrides go infrared: new properties and perspectives, J. Appl. Phys. 106 (2009) 011101

[5] N.H. Alvi, P.E.D. Soto Rodriguez, V.J. Gómez, P. Kumar, G. Amin, O. Nur, M. Willander, R. Nötzel, Highly efficient potentiometric glucose biosensor based on functionalized InN quantum dots, Appl. Phys. Lett. 101 (2012) 153110.

[6] N.H. Alvi, P.E.D. Soto Rodriguez, P. Aseev, V.J. Gómez, A.H. Alvi, W. Hassan, M. Willander, R. Nötzel, InN/InGaN quantumdotphotoelectrode: efficient hydrogen generation by water splitting at zero voltage, Nano Energy 13 (2015) 291-297.

[7] P. Aseev, P.E.D. Soto Rodriguez, V.J. Gómez, N.H. Alvi, J.M. Mánuel, F.M. Morales, J.J. Jiménez, R. García, A. Senichev, C. Lienau, E. Calleja, R. Nötzel, Near-infrared emitting In-rich InGaN layers grown directly on Si: Towards the whole composition range, Appl. Phys. Lett. 106 (2015) 072102.
[8] P.E.D. Soto Rodriguez, P. Aseev, V.J. Gómez, P. Kumar, N.H. Alvi, E. Calleja, J.M. Manuel, F.M. Morales, J.J. Jiménez, R. García, A. Senichev, C. Lienau, R. Nötzel, Stranski-Krastanov InN/InGaN quantum dots grown directly on Si(111), Appl. Phys. Lett. 106 (2015) 023105.

[9] P.E.D. Soto Rodriguez, P. Kumar, V.J. Gómez, N.H. Alvi, J.M. Manuel, F.M. Morales, J.]. Jiménez, R. García, E. Calleja, R. Nötzel, Spontaneous formation of InGaN nanowall network directly on Si, Appl. Phys. Lett. 102 (2013) 173105.

[10] N.H. Alvi, P.E.D. Soto Rodriguez, V.J. Gómez, P. Aseev, A.H. Alvi, W.M.A. Alvi, M. Willander, R. Nötzel, Photoelectrochemical water splitting and hydrogen generation by a spontaneously formed InGaN nanowall network, Appl. Phys. Lett. 104 (2014) 223104.

[11] C.G. Van de Walle, D. Segev, Microscopic origins of surface states on nitride surfaces, J. Appl. Phys. 101 (2007) 081704.

[12] Y.L. Chang, Z. Mi, F. Li, Photoluminescence properties of a nearly intrinsic single InN nanowire, Adv. Funct. Mater. 20 (2010) 4146-4151.

[13] W.M. Linhart, T.D. Veal, P.D.C. King, G. Koblmüller, C.S. Gallinat, J.S. Speck, C.F. McConville, Surface, bulk, and interface electronic properties of nonpolar InN, Appl. Phys. Lett. 97 (2010) 112103.

[14] N. Sofikiti, G. Tsiakatouras, E. Iliopoulos, A. Georgakilas, N. Chaniotakis, Influence of crystal polarity and metal electronegativity on the sensing properties of III-nitrides, J. Mater. Sci. Eng. 3 (2009) 28-39.

[15] N. Sofikiti, N. Chaniotakis, J. Grandal, M. Utrera, M.A. Sánchez-García, E. Calleja, GaN and InN nanocolumns as electrochemical sensing elements: potentiometric response to KCl, pH and urea, Mater. Lett. 64 (2010) 1332-1335.

[16] L.R. Bailey, T.D. Veal, P.D.C. King, C.F. McConville, J. Pereiro, J. Grandal, M.A. SánchezGarcía, E. Muñoz, E. Calleja, Band bending at the surfaces of In-rich InGaN alloys, J. Appl. Phys. 104 (2008) 113716. 